\title{
MÉTODOS CONSTRUTIVOS DE BARRAGENS DE REJEITOS DE MINERAÇÃO - UMA REVISÃO
}

\author{
F. A. C. CARDOZO*, M. M. PIMENTA e A. C. ZINGANO
Universidade Federal do Rio Grande do Sul
fernando.cantini3@gmail.com \\ Artigo submetido em 21/11/2016 e aceito em 14/12/2016 \\ DOI: $10.15628 /$ holos.2016.5367
}

\section{RESUMO}

Historicamente no Brasil a indústria de extração mineral é responsável por grande porção da economia nacional. Um grande desafio enfrentado pelo setor mineral é a disposição de rejeitos, oriundos das fases de lavra e/ou beneficiamento. Estes materiais são dispostos normalmente em duas modalidades: pilhas de estéril e/ou barragem de rejeitos. Atualmente são discutidos os problemas relacionados com a disposição em barragem de rejeitos, as quais em caso de falha, são potenciais causadoras de grandes prejuízos ambientais, econômicos e sociais. Diversos casos de acidentes em barragens de mineradoras no Brasil endossam os riscos
\end{abstract}

associados à falha das mesmas, os quais motivaram este estudo. Um ponto peculiar no estudo e gerenciamento das barragens de mineração é o caráter multidisciplinar visto a necessidade de sinergia entre diferentes áreas, como geotécnica, processamento mineral, planejamento de produção, entre outras. Este trabalho se propõem a analisar os riscos associados aos tipos de método construtivos de barramento. Para tanto, são abordados os três métodos construtivos conhecidos: método de alteamento à jusante, de alteamento à montante e da linha de centro, os quais são revisados.

PALAVRAS-CHAVE: Barragem de rejeitos, Métodos construtivos, Mineração

\section{CONSTRUCTION METHODS OF MINING TAILINGS DAM}

\begin{abstract}
Historically in Brazil the mining industry is responsible for a large portion of the national economy. A major challenge faced by the mining industry is the waste disposal management, resulting from the stages of mining production and/or mineral processing. The waste management is usually stored in two ways: mine-waste pile and/or tailings dam. Currently the problems related to the tailings dam disposal are widely discussed, which in case of failure, are potential cause of major environmental, economic and social damage. Several cases of accidents in mining tailings dam in Brazil
\end{abstract}

endorse the risks associated with their failure, which have motivated this study. A peculiar point in the study and management of surface retaining structures is the multidisciplinary, and its necessity for synergy between different fields of engineering, such as geotechnics, mineral processing, mine planning, among others. This work proposes to analyze the risks associated with the different constructive methods of tailings dam. Thereunto, the main constructive methods have been extensively reviewed: the downstream method, the upstream method and the centerline method.

KEYWORDS: Tailings Dam, Construction Methods, Mining 


\section{INTRODUÇÃO}

Juntamente com pilhas de estéril as barragens são as maiores estruturas geotécnicas construídas pelo homem. Barragens são construídas pela humanidade há milênios para diversos fins, como: armazenamento de água, controle de vazões, geração de energia hidrelétrica, entre outros. Com o avanço da exploração mineral e a necessidade de conservação socioambiental relacionada com a mesma, o assunto barragem de rejeitos vem merecendo grande atenção, devido a seu potencial danoso em caso de falhas. Historicamente vemos com certa frequência a ruptura de barragens de mineração e os danos causados por estas. Azam e Li (2010), apresentam um histórico mundial das falhas em barragens de mineração, onde segundo a revisão dos autores cerca de $1,2 \%$ das barragens de mineração apresentaram algum tipo de falhas, contra 0,01\% das barragens civis, isto para os 100 anos antecessores à publicação.

Recentemente o Brasil passou pelo o que é considerado o seu mais grave acidente ambiental em decorrência do rompimento da Barragem de Rejeitos de Fundão, em Mariana/ MG (CETEM, 2015). Tal opinião ainda é compartilhada por Lopes (2016), quanto a escala dos danos decorrentes da ruptura do barramento. Salientando que, segundo relatório de Morgenstern, Vick, Viotti, e Watts (2016) a referida barragem era constituída predominantemente por alteamento pelo método de montante e uma parte pelo método de linha de centro.

Visto o potencial danoso das barragens de rejeito, este trabalho propõe-se a lançar luz ao tema e apontar alguns pontos relevantes quanto as propriedades e métodos construtivos de barragem de rejeitos. Em virtude da multidisciplinaridade requerida nas fases de projeto, execução e operação, assim englobando diversos profissionais de engenharia de minas e geotécnica e áreas das ciências de mecânica dos solos e das rochas. Fatores estes que também distinguem as barragens de rejeitos de mineração (Figura 1 ) de demais obras geotécnicas e das ditas barragens civis.

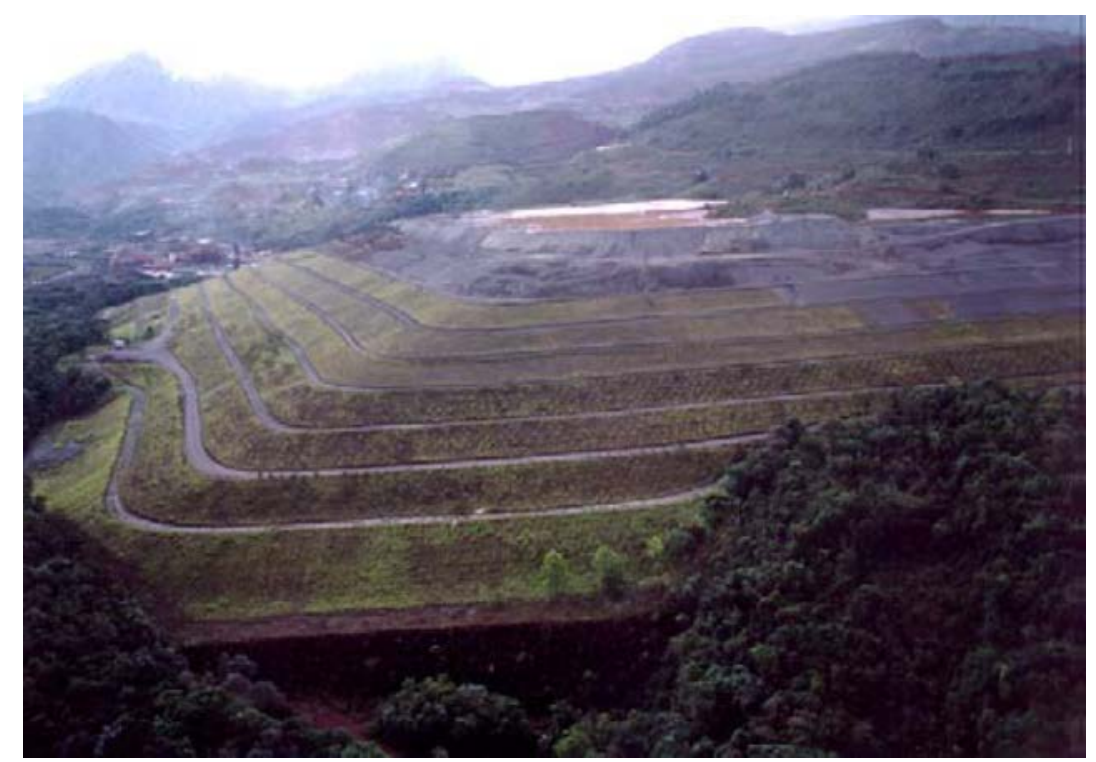

Figura 1: Barragem construída a Montante (Fonte: Espósito (2000) apud. Figueiredo (2007)) 


\section{BARRAGEM NA MINERAÇAO}

São muitos fatores que diferenciam as barragens de rejeitos oriundas da mineração de barragens para obras civis em geral. Duarte (2008) salienta que barragens civis ou convencionais tem qualquer fim menos a contenção de rejeitos. Outro ponto que diferencia as barragens de rejeito é a sua construção em etapas, as quais, acompanham o ritmo de lavra, desta forma à medida que são gerados rejeitos, os alteamentos são executados. Estas, também, são reconhecidas por gerarem um impacto ambiental significante, eventuais falhas resultam em grandes volumes de rejeitos descarregados no meio ambiente (Duarte, 2008).

Diferente de barragens convencionais, que normalmente barram água, barragens de rejeito armazenam rejeitos dos processos minerais que podem variar de materiais arenosos não plásticos (rejeitos granulares) até solos de granulometria fina e alta plasticidade (lamas) (Araújo, 2006). Kossoff et al. (2014), define os rejeitos destinados a barragens como mistura de rocha cominuída e os fluidos do processo de beneficiamento; então apresentam como característica física: granulometria fina e forma angular; e composição química dependente da composição da rocha de origem e dos reagentes utilizados no processamento mineral.

Este material por ter passado por processos de beneficiamento, no qual normalmente envolve reações químicas, apresentam composições iônicas variadas. As quais além de por vezes serem nocivas ao homem e meio ambiente são de um ponto de vista construtivo negativas pela possibilidade de interação e reação química com os materiais construtivos do aterro, assim alterando a permeabilidade. Davies, Lighthall, Rice e Martin (2002), em um amplo trabalho de revisão sobre barragens de rejeito, salienta a necessidade de consideração da relação geoquímica dos rejeitos.

Também ressalta-se a necessidade de ações sinérgicas entre diversos profissionais, no caso específicos de barragem de rejeitos, entre engenharia geotécnica, a qual é a responsável pelo projeto e execução da obra, com a engenharia de processos, a qual é a responsável pelo beneficiamento do minério e também diretamente responsável pelas características geotécnicas do material a ser depositado e da taxa de deposição (Chaves, 1998).

\section{MÉTODOS CONSTRUTIVOS}

Basicamente existem três metodologias construtivas para barragens de rejeito: (i) Método da jusante, (ii) Método de montante e (iii) Método da linha de centro.

\subsection{Método da jusante}

Consiste de construção e alteamento do barramento sempre a jusante. Na Figura 2 vemos um esquema simplificado de uma barragem à jusante e na Figura 3 vemos os alteamentos posteriores. 


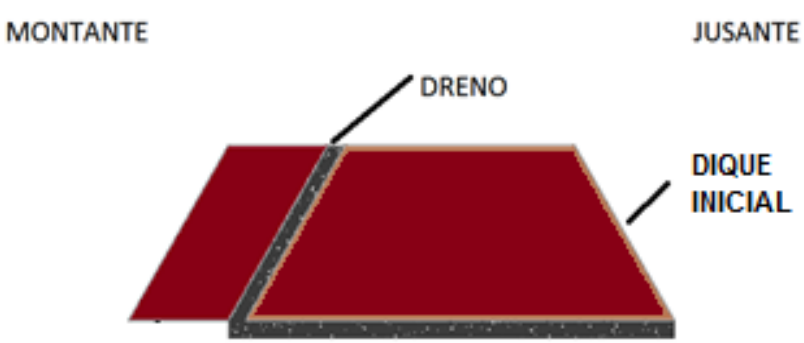

Figura 2: Estrutura inicial de uma barragem a jusante

Na figura 3 é apresentado os alteamentos posteriores.

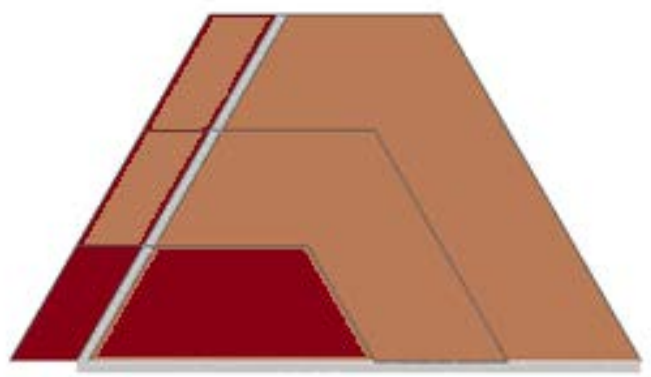

Figura 3: Barramento a Jusante

Chammas (1989 como citado em Passos, 2009, p. 21) comenta que as vantagens do método de jusante estão na sua resistência a carregamentos dinâmicos, isto deve-se ao fato de escalonar a construção sem interferir na segurança, dessa forma facilita a drenagem, possui baixa susceptibilidade de liquefação e simplicidade na operação.

\subsection{Método da montante}

Consiste de construção e alteamento do barramento sempre à montante sobre o rejeito já consolidado. Na Figura 4 é apresentado a estrutura inicial de um barramento à montante, com o dique inicial e a praia de rejeito.

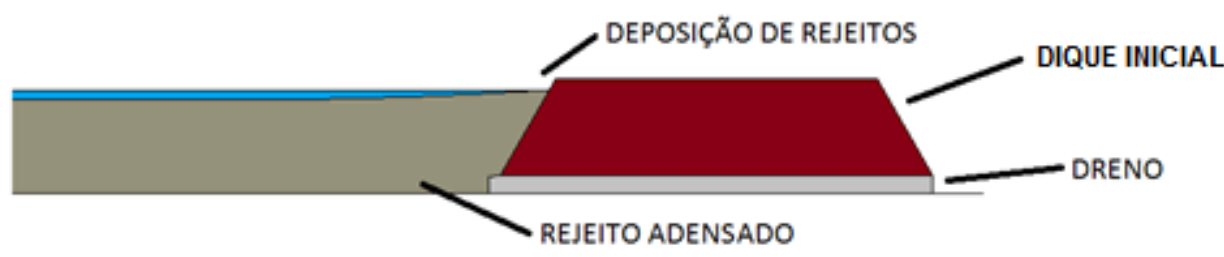

Figura 4: Estrutura inicial do barramento a Montante

Na Figura 5 abaixo, está representado os posteriores alteamentos realizados sob o rejeito. 


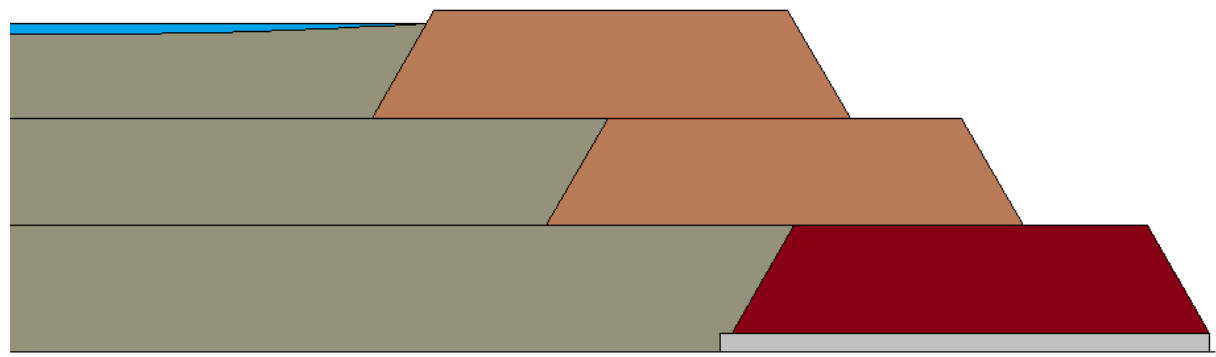

Figura 5: Barramento a Montante

Embora este seja o mais utilizado pela maioria das mineradoras, o método de montante apresenta um baixo controle construtivo tornando-se crítico principalmente em relação à segurança (Araújo, 2006). Segundo Martin e McRobert (1999) o método de alteamento à montante representa um desafio no âmbito geotécnico devido às tensões induzidas, potencial de liquefação e não consolidação do material utilizado como fundação.

\subsection{Método linha de centro}

Consiste de construção e alteamento do barramento tanto à montante quanto à jusante, acompanhando um eixo vertical, chamado de linha de centro, sobre o rejeito depositado a montante e sobre o próprio barramento à jusante. O método da linha de centro é um método intermediário que tenta minimizar as desvantagens entre o método de montante e o de jusante (Passos, 2009).

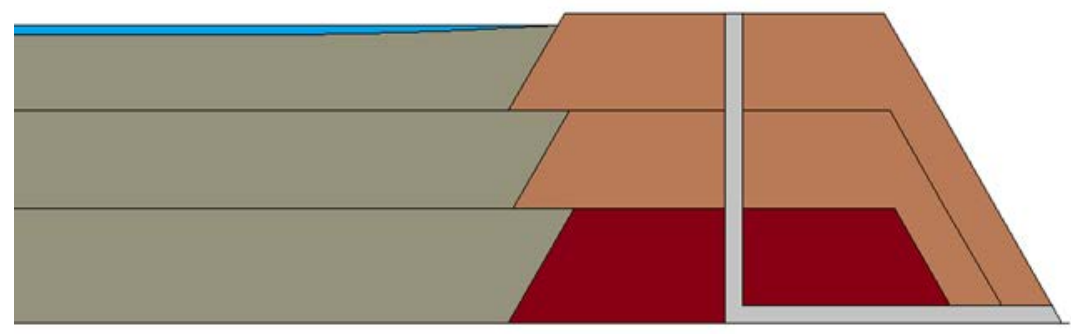

Figura 6: Barramento pelo método de Linha de Centro

\subsection{Sistema de drenagem}

Os drenos, estruturas comuns a todos os métodos construtivos, são responsáveis por prevenir excessos de poro pressão devido ao fluxo de água da barragem. As forças de poro pressão são forças favoráveis ao movimento. Caso não haja eficiência no sistema de drenagem em direcionar o fluxo na barragem, podemos ter a ocorrência do fenômeno chamado piping, definido como a erosão interna que provoca a remoção de partículas do interior do solo, formando "tubos" vazios que provocam colapsos e escorregamentos laterais do terreno. Este fenômeno é descrito por Marques Filho e Geraldo (2002), ocorrendo em solos pouco coesos onde o gradiente hidráulico é maior que a coesão do solo. 
Rafael e Romanel (2014) concluem que a velocidade de disposição de rejeitos e de alteamentos junto com drenagem ineficiente contribuem para ocorrência de piping em barragem a montante. O que podemos expandir para os demais métodos, considerando, o sistema de drenagem comum a todos os métodos e os alteamentos subsequentes.

\subsection{Comparativo entre os métodos}

A Tabela 1 a seguir apresenta um resumo comparativo entre os principais métodos construtivos de barragens de rejeitos, destacando suas vantagens, desvantagens, características, propriedades, entre outros.

Tabela 1: Resumo comparativo dos principais métodos construtivos de barragens de rejeito

\begin{tabular}{c|c|c|c}
\hline Tipo de rejeito & Montante & Jusante & Linha de centro \\
\hline $\begin{array}{c}\text { Baixa densidade para que } \\
\text { ocorra segregação }\end{array}$ & Qualquer tipo & $\begin{array}{c}\text { Areias de lamas } \\
\text { de baixa } \\
\text { plasticidade }\end{array}$ \\
\hline $\begin{array}{c}\text { Armazenamento de } \\
\text { água }\end{array}$ & $\begin{array}{c}\text { Não recomendável para } \\
\text { grandes volumes }\end{array}$ & Independe & Periférica \\
\hline $\begin{array}{c}\text { Resistência a abalos } \\
\text { sísmicos }\end{array}$ & Baixa & Boa & Aceitável \\
\hline Alteamentos & Ideal menos 10 m/ano & Nenhuma restrição & Pouca restrição \\
\hline $\begin{array}{c}\text { Vantagens } \\
\text { onde há restrição de área }\end{array}$ & Maior segurança & $\begin{array}{c}\text { Flexibilidade } \\
\text { construtiva }\end{array}$ \\
\hline Desvantagens & $\begin{array}{c}\text { Baixa segurança } \\
\text { suscetibilidade a } \\
\text { liquefação e piping }\end{array}$ & $\begin{array}{c}\text { Grande quantidade de } \\
\text { material requerido proteção } \\
\text { do talude a jusante apenas } \\
\text { na configuração final }\end{array}$ & $\begin{array}{c}\text { Necessidade de } \\
\text { eficiente sistema } \\
\text { de drenagem }\end{array}$ \\
\hline
\end{tabular}

\section{PRINCIPAIS PROBLEMAS ASSOCIADOS AO MÉTODO CONSTRUTIVO}

Devido ao método construtivo e a dinâmica empregada na mineração o método que tende a apresentar menos problemas é o método de jusante. Isto devido ao fato de a geometria da barragem apresentar certa constância e o controle das propriedades dos materiais de construção independe do ritmo de deposição do rejeito, ou seja, se tem um controle nítido de toda a barragem, compactação do material, drenos e impermeabilização.

Por ser construída sobre o material de rejeito depositado, o método de montante nos leva a uma maior dificuldade de controle das propriedades geotécnicas da zona em que é realizada o alteamento, ou seja, rejeito adensado. De acordo com Araújo (2006) e Martin e McRobert (1999), pelo fato dos alteamentos serem realizados sobre materiais previamente depositados e não consolidados, estes estão em condição saturada e tendem a apresentar baixa resistência ao cisalhamento e susceptibilidade à liquefação por carregamentos dinâmicos e estáticos. Araújo (2006), também comenta os problemas decorrentes do sistema de drenagem interno referentes a este método. 
Quanto ao método de linha de centro, classifica-se como sendo um método intermediário, com mais riscos associados que o método de jusante e menos que o de montante. Quanto a ele destaca-se o fato de que o dreno está localizado em posição fixa, vertical, e metade do alteamento ocorre pelo método de alteamento a jusante, o que leva aos pontos positivos deste método.

Como respaldo ao supracitado temos o estudo de Rico, Benito, Salgueiro, Díez-Herrero e Pereira (2008), onde este apresenta a relação de falhas em barragens em relação ao método construtivo. Onde o método de montante corresponde a $76 \%$ das falhas em barragem, sendo 0 restante (24\%) proveniente dos demais métodos construtivos (jusante, linha de centro e outros).

Quanto a análise de estabilidade da barragem, temos uma relação nítida da execução da mesma com a geometria e disposição dos materiais e suas propriedades. Ou seja, quanto mais simples a geometria da barragem e mais homogênea for a distribuição dos materiais envolvidos, mais facilitado será o processo de análise da estabilidade, seja por métodos numéricos ou analíticos (Cardozo, Cordova, Zingano, Galli e Peña, 2016).

O método de jusante apresenta um procedimento de análise mais simples frente aos demais, sobretudo ao método de montante. O método de montante apresenta uma geometria e disposição de materiais em camadas alternadas que dificulta sua análise analítica e computacional, fazendo-se necessário, por exemplo para uma análise numérica um modelo refinado com camadas de geometria e propriedades alternadas.

Quanto as análises a serem realizadas, o que há de mais atual são os modelos em elementos finitos, os quais fazem a análise mesclando hipóteses de deslizamento, tombamento e ruptura circular. Quanto ao mecanismo mais frequente de ruptura, Marques Filho e Geraldo (2002) apontam o deslizamento em eventuais planos de fraqueza da fundação, devido ao mau controle construtivo.

Para a análise geotécnica Rafael e Romanel (2014), apresentam metodologia útil a ser seguida para retroanálise em barragem onde correlacionam os valores de SPT (Standard Penetration Test) para inferir parâmetros geotécnicos dos materiais, os quais seriam de difícil aquisição direta por serem necessários ensaios laboratoriais em amostras indeformadas, ou seja, sob condições especificas. Quanto à inferência de parâmetros geotécnicos pelo ensaio de SPT, Schnaid (2000) apresenta diversas metodologias de associações de parâmetros geotécnicos com os valores obtidos por este ensaio.

\section{CONCLUSÃO}

Analisando as opções de método construtivo de barragens de rejeito, conclui-se que o método de montante perde em confiabilidade para o método de jusante. Diversos acidentes são associados ao método de montante, este amplamente empregado na mineração brasileira. Visto isto, fica nítido a necessidade de aprofundamento em estudos que levem a uma maior utilização do método de jusante no Brasil.

Talvez seja necessária uma mudança ideológica no setor mineral. Onde os riscos referentes a falha em barragem sejam mais estudados para que se pondere quanto ao desprendimento de recursos para investigação geotécnica, construção, operação e manutenção de barragens de rejeito. Segundo Davies et al. (2002), os custos diretos provenientes de uma 
falha de barragem são da ordem de milhões de dólares, sendo que os custos indiretos são ainda muito superiores a esses. Isto mostra e evidencia a necessidade de estudos aprofundados da metodologia construtiva a ser adotada e da análise de estabilidade previa e concomitante a operação.

Conclui-se por fim, que o método de montante, utilizado amplamente na mineração, apresenta maior propensão a falha por ser um método que apresenta complexidade de controle executivo. Por tanto, propõem-se utilização do método de jusante, por embora apresentar maior custo construtivo e operacional, ser um método onde tem-se um melhor controle dos materiais envolvidos e de suas propriedades.

\section{REFERÊNCIAS}

1. Araújo, C. B. (2006). Contribuição ao estudo do comportamento de barragens de rejeito de mineração de ferro. Tese de Doutorado, Universidade Federal do Rio de Janeiro, Rio de Janeiro, RJ, Brasil.

2. Azam, S., \& Li, Q. (2010). Tailings dam failures: A review of the last one hundred years. Geotechnical News, 28(4), 50-54.

3. Cardozo, F. A. C., Cordova, D. P., Zingano, A. C., Galli, B., \& Penã, F. P. (2016). Métodos Construtivos de Barragens de Rejeito: Crítica ao Método de Montante. Anais do Congresso Brasileiro de Geologia, Porto Alegre, RS, Brasil, 48.

4. CETEM. (2015). Rompimento de barragem de rejeitos em Mariana (MG) é o maior acidente ambiental da História do Brasil. Recuperado em 12 de dezembro, 2016, de http://verbetes.cetem.gov.br/verbetes/ExibeVerbete.aspx?verid=213

5. Chaves, A. P (1996). Teoria e prática do tratamento de minérios. Volumes 1. São Paulo: Signus Editora.

6. Davies, M. P., Lighthall, P. C., Rice, S., \& Martin, T. E. (2002). Design of tailings dams and impoundments. Keynote address, Tailings and Mine Waste Practices SME, AGM Phoenix.

7. Duarte, A. P. (2008). Classificação das barragens de contenção de rejeitos de mineração e de resíduos industriais no estado de Minas Gerais em relação ao potencial de risco. Dissertação de Mestrado, Universidade Federal de Minas Gerais, Belo Horizonte, MG, Brasil.

8. Figueiredo, M. M. (2007). Estudo de metodologias alternativas de disposição de rejeitos para a mineração Casa de Pedra-Congonhas/MG. Dissertação de Mestrado, Universidade Federal de Ouro Preto, Ouro Preto, MG, Brasil.

9. Kossoff, D., Dubbin, W. E., Alfredsson, M., Edwards, S. J., Macklin, M. G., \& Hudson-Edwards, K. A. (2014). Mine tailings dams: characteristics, failure, environmental impacts, and remediation. Applied Geochemistry, 51, 229-245.

10. Lopes, L. M. N. (2016). O rompimento da barragem de Mariana e seus impactos socioambientais. Sinapse Múltipla, 5(1), 1-14.

11. Martin, T. E., \& McRoberts, E. C. (1999). Some considerations in the stability analysis of upstream tailings dams. In Proceedings of the Sixth International Conference on Tailings and Mine Waste, Vol. 99, 287-302.

12. Marques Filho, P. L., \& Geraldo, A. (1998). Barragens e Reservatórios. In: A. M. dos S. Oliveira 
\& S. N. A. de Brito (Ed.). Geologia de Engenharia. São Paulo: ABGE.

13. Morgenstern, N. R., Vick, S. G., Viotti, C. B., \& Watts, B. D. (2016) Fundão Tailings Dam Review Panel. Retrieved December 13, 2016, from http://fundaoinvestigation.com/wpcontent/uploads/general/PR/en/FinalReport.pdf

14. Passos, N. C. S. T. (2009). Barragem de Rejeito: Avaliação dos Parâmetros Geotécnicos de Rejeito de Minério de Ferro utilizando Ensaios de Campos-Um Estudo de Caso. Trabalho de Conclusão de Curso Universidade Federal do Paraná, Curitiba, PR, Brasil.

15. Rafael, H. M. A. M.\& Romanel, C. (2014). Potencial de Liquefação Estática no Alteamento de uma Barragem de Rejeito. Anais do Congresso brasileiro de Mecânica de Solos e Engenharia Geotécnica, Goiânia, GO, Brasil, 14.

16. Rico, M., Benito, G., Salgueiro, A. R., Díez-Herrero, A., \& Pereira, H. G. (2008). Reported tailings dam failures: a review of the European incidents in the worldwide context. Journal of Hazardous Materials, 152(2), 846-852.

17. Santos, D. A. M. dos; Curi, A., \& Silva, J. M. da (2014). Técnicas para a disposição de rejeitos de minério de ferro. Anais do Congresso brasileiro de Mina a Céu Aberto e de Mina Subterrânea, Belo Horizonte, MG, Brasil, 8.

18. Schnaid, F (2000). Ensaios de Campo e suas aplicações na engenharia de fundações. São Paulo: Oficina de Textos. 